\title{
CONCEITOS DE RECOVERY E TRABALHO PARA PESSOAS EM SOFRIMENTO MENTAL
}

\section{Recovery Concepts and Employment for People with Serious Mental Illness}

\author{
Mark Napoli Costa, M.D., MPH${ }^{1}$ \\ Graziela do Carmo Reis, BS, MPH² \\ Ellen Ricci, Ph.D. ${ }^{3}$
}

Resumo: $O$ processo de Reforma Psiquiátrica no Brasil, nos últimos 30 anos, produziu avanços na qualidade e no acesso ao cuidado de pessoas com sofrimento mental que são impressionantes. Uma rede de atenção psicossocial robusta e efetiva foi construída e consolidada. A Atenção Psicossocial sustenta em teoria e prática mudança nos cuidados em saúde mental em toda a rede do Sistema Único de Saúde (SUS). O presente artigo, tem por objetivo promover um diálogo entre as abordagens inovadoras na atenção psicossocial e os conceitos, estratégias e práticas em saúde mental orientadas por Recovery. Estratégias desenvolvidas pela Atenção Psicossocial irão dialogar com a teoria que sustenta práticas e abordagens inovadoras orientadas por Recovery, tais como suporte de pares, gestão autônoma da medicação (GAM) suporte para o trabalho e renda de pessoas com experiência vivida em saúde mental e instrumento de avaliação de serviços orientados ao Recovery.

Palavras-Chave: Atenção Psicossocial, Recovery, Saúde Mental, Pessoas com Experiência Vivida.

Abstract: The Psychiatric Reform process in Brazil, in the last 30 years, has produced impressive advances in the quality and access to care for people with mental suffering. A robust and effective psychosocial care network was built and consolidated. Psychosocial care sustains in theory and practice changes in mental health care throughout the Unified Health System (SUS) network. This article aims to promote a dialogue between innovative approaches in psychosocial care and the concepts, strategies, and practices in mental health

\footnotetext{
${ }^{1}$ Médico, Mestre em Saúde Pública, Pesquisador associadot, Program for Recovery and Community Health (PRCH), Department of Psychiatry, Yale School of Medicine. mark.costa@yale.edu

2 Psicóloga, mestra em Saúde Pública. Program for Recovery and Community Health (PRCH), Department of Psychiatry, Yale School of Medicinfe. graziela.reis@yale.edu

${ }^{3}$ Doutora em Saúde Coletiva, Docente do Curso de Terapia Ocupacional, Faculdade de Medicina, Universidade Federal de Pelotas - RS, Brasil. ellenricci@gmail.com
} 
guided by Recovery. Strategies developed by Brazilain psychosocial care will dialogue with the theory that supports Recovery. Innovations include peer support, autonomous medication management (GAM), support employment for people with lived experience in mental health, and an evaluation instrument of recovery-oriented services.

Keywords: Psychosocial Care, Recovery, Mental Health, People with Lived Experience

\section{Introdução}

"Devemos, portanto, garantir a participação da comunidade. Sem isso não é possível ir mais longe nessa direção [...]. Como essa participação pode ser alcançada é um problema para o futuro ..." (BASAGLIA, 1980, p. 27). O processo de Reforma Psiquiátrica no Brasil, nos últimos 30 anos, produziu avanços na qualidade e no acesso ao cuidado de pessoas com sofrimento mental que são impressionantes (CAMPOS; COSTA; PEREIRA; RICCl et al., 2017; COSTA, 2016; ONOCKO-CAMPOS; PASSOS; PALOMBINI ADE; DOS SANTOS et al., 2013; RICCl; CRISTINA; LEAL; MARIA et al., 2021; RICCI; LEAL; LA-ROTTA; ONOCKO-CAMPOS et al., 2020). Uma rede de atenção psicossocial robusta e efetiva foi construída e consolidada. A Atenção Psicossocial sustenta em teoria e prática mudança nos cuidados em saúde mental em toda a rede do Sistema Único de Saúde (SUS). Na passagem acima, Franco Basaglia antecipa o que viria a se tornar um desafio central da próxima fase da transformação ou inovação do sistema de saúde mental (DAVIDSON, 2017). Desafios estruturais, econômicos, tecnológicos e principalmente o desafio de oferecer um serviço centrado em cuidados que respeitam e valorizam o querer, a potencialidade, o direito de ter uma vida plena das pessoas com história de vida em saúde mental (COSTA, 2017). Por ter nascido essencialmente dos movimentos de direitos humanos e sociais nos Estados Unidos e Europa, o movimento Recovery reconhece, de uma maneira bem ampla, o direito de pessoas diagnosticadas ou manifestando sintomas de um sofrimento mental (mesmo que considerado severo) de participar plenamente da sociedade (COSTA, 2017; DAVIDSON, 2016).

\section{Sobre o Recovery}


Recovery refere-se à experiência de vida real vivida pelas pessoas em sofrimento mental. Recovery não é um processo de normatização ou normalização de pessoas em sofrimento mental (DEEGAN, 1996). As coisas que uma pessoa faz para minimizar o que é percebido como doença são diferentes do que se faz para maximizar uma vida de significado. Não se trata de reabilitar uma pessoa que ela possa retornar à sociedade com sucesso (reabilitação psicossocial). Trata-se da construção de um senso de identidade que vai além dos limites da doença.Não se trata de curar, reduzir sintomas ou adquirir percepções e habilidades, mas possibilitar que pessoas em sofrimento mental possam viver plenamente independente da intensidade dos sintomas ou das habilidades que a pessoa possui ou deseja possuir.

Viver plenamente implica necessariamente a comunidade onde a pessoa vive. O que exclui as pessoas em sofrimento mental da vida na comunidade éa maneira como são vistas por outras pessoas e os processos de discriminação e preconceito contra pessoas em sofrimento mental.

No cuidado orientado pelo Recovery, inclusão social é visto como um direito e não como mérito, consequência ou recompensa de um processo de reabilitação ou tratamento. Pessoas em sofrimento mental primeiramente têm o direito a uma vida na comunidade, ou seja, direito à moradia, trabalho, independência financeira, relacionamento, autodeterminação, cidadania. Autodeterminação é um direito que não pode ser minimizado ou negligenciado e inclui decisão sobre o próprio tratamento, incluindo decisão sobre uso de medicamentos.

Várias estratégias de Recovery vêm sendo incorporadas no cuidado em saúde mental. Assim, o suporte de pares, o tratamento centrado na pessoa, a decisão compartilhada, os grupos de escutadores de vozes, e estratégias de cidadania, entre outras, começam a fazer parte do cotidiano dos serviços substitutivos ao modelo centrado no hospital, nos diversos países do mundo.

\section{Recovery e Direitos}


Vários países buscaram a aprovação de leis para reafirmar e garantir direitos específicos de pessoas em sofrimento mental. Para tal, um caminho comum foi reconhecer o sofrimento mental como uma desabilidade. Nos Estados Unidos, este foi o caminho escolhido pelos movimentos sociais de pessoas em sofrimento mental, como o movimento dos Sobreviventes da Psiquiatria. Ao longo da década de 80 , os diversos movimentos sociais de pessoas com desabilidades se organizaram e passaram discutir com o congresso a aprovação de uma lei reafirmando que possuir uma desabilidade física ou mental de maneira alguma diminui o direito desta pessoa em participar de todos os aspectos da sociedade. A lei americana aprovada em 1990 (Americans with Disabilities Act - ADA 1990) reconhece também que o preconceito e discriminação contra pessoas com desabilidades estão no cerne das barreiras institucionais e outras estruturas sociais que excluem pessoas com desabilidades da participação plena de todos os aspectos da vida comunitária. A lei vai então, entre outras coisas, proibir toda forma de discriminação contra estas pessoas. Ela afirma também que cabe à sociedade construir formas para acomodar as diversas desabilidades para permitir que pessoas portadoras de desabilidade possam viver a vida que desejam.

\section{A Lei das Pessoas com Desabilidades e o Trabalho}

Acesso a moradia, independência financeira, vida na comunidade, e o acesso ao trabalho competitivo e integrado são alguns dos instrumentos disponíveis para empoderar pessoas em sofrimento mental na construção de uma vida de significado, ou seja, no seu processo de Recovery. Barreiras institucionais que dificultam ou inviabilizam o acesso a estes instrumentos devem ser derrubadas. O trabalho é um exemplo emblemático para entendermos como a discriminação e o preconceito ajudam a erguer estas barreiras.

Diversos estudos realizados em diferentes países do mundo demonstram claramente que entre 60 e $70 \%$ das pessoas em sofrimento mental querem trabalhar. Além de propiciar renda, o trabalho promove bem estar mental, facilita a participação na comunidade, e ajuda a desenvolver um papel social de valor para a pessoa em sofrimento mental (SHANKAR; LI; TAN, 2021). Fica claro, então, que o emprego competitivo (emprego como outro qualquer) e integrado (onde a pessoa com está integrada no ambiente de trabalho e se sente confortável 
com os outros colegas trabalhadores) pode ser um instrumento de valor para muitos no seu Recovery. No Brasil existem diversas barreiras institucionais que tornam praticamente impossível que uma pessoa em sofrimento mental consiga um trabalho competitivo e integrado, impedindo o acesso a um instrumento de grande valor no processo de Recovery.

Países que adotaram leis contra a discriminação de pessoas em sofrimento mental reconhecem por um lado que o sofrimento mental pode ser uma desabilidade e que a discriminação de pessoas portadoras de desabilidade é ilegal. Isso vai permitir que estas pessoas possam advogar pelo reconhecimento da sua desabilidade quando conveniente, sabendo que a lei vai proteger em caso de discriminação. O empoderamento é fundamental no processo de Recovery.

Assim, utilizando o trabalho como um exemplo, nos Estados Unidos nenhum empregador pode pedir um atestado médico ou exame de sanidade mental no processo de admissão. Qualquer pergunta referente a tratamento de saúde mental no processo admissional pode ser entendida como discriminatória e o empregador pode ser processado. A informação de saúde é privada e ninguém pode acessar a informação de saúde de outra pessoa sem o explícito consentimento. Nenhum histórico de trabalho contém informação de saúde e muito menos o histórico de afastamento por motivo de doença. O empregador não pode acessar o banco de dados da previdência social para saber do histórico previdenciário de um candidato ou empregado. Por outro lado, a pessoa em sofrimento mental que está trabalhando ou cursando uma universidade pode exigir acomodações especiais para a sua desabilidade, amparada pela lei. Assim, um trabalhador pode demandar trabalhar em um ambiente mais sossegado, ou ter intervalos mais frequentes para acomodar a sua desabilidade.Um estudante pode pedir um prazo maior para concluir uma prova ou terminar um trabalho, como forma de acomodação da sua desabilidade. Atualmente existem várias pesquisas em curso buscando entender quais as melhores formas de acomodar as diversas formas de desabilidade no trabalho.

\section{Conclusão}

Na busca de estratégias e instrumentos para ajudar pessoas em sofrimento mental no seu processo singular de Recovery, o amparo legal é fundamental para empoderar pessoas 
em sofrimento mental na busca da vida que desejam, em suas comunidades. $O$ reconhecimento do sofrimento mental como uma desabilidade foi a forma que diversos países encontraram para construir leis proibindo a discriminação de pessoas com desabilidades e facilitando a inclusão de pessoas com desabilidade em suas comunidades através do trabalho, moradia, renda etc. Estas leis também servem de base para avançar políticas de cuidado no campo da saúde mental como o suporte de pares, o tratamento centrado na pessoa, o grupo de escutadores de vozes, a decisão compartilhada e as estratégias de cidadania, entre outras. Estas estratégias podem reforçar e ajudar a avançar a luta antimanicomial no Brasil e no mundo.

REFERÊNCIAS:

CAMPOS, R. T. O.; COSTA, M.; PEREIRA, M. B.; RICCI, E. C. et al. Recovery, citizenship, and psychosocial rehabilitation: A dialog between Brazilian and American mental health care approaches. American Journal of Psychiatric Rehabilitation, 20, n. 3, p. 311-326, 2017.

COSTA, M. Stakeholder involvement in forensic psychiatry: The Brazilian experience. The journal of the American Academy of Psychiatry and the Law, 44, n. 1, p. 2, 2016.

COSTA, M. N. Recovery como estratégia para avançar a reforma psiquiátrica no Brasil. Cadernos Brasileiros de Saúde Mental/Brazilian Journal of Mental Health, 9, n. 21, p. 01-16, 2017.

DAVIDSON, L. The recovery movement: Implications for mental health care and enabling people to participate fully in life. Health Affairs, 35, n. 6, p. 1091-1097, 2016.

DAVIDSON, L. After the asylum: A Basaglian-informed visin of Recovery-oriented care. Cadernos Brasileiros de Saúde Mental/Brazilian Journal of Mental Health, 9, n. 21, p. 125-136, 2017.

DEEGAN, P. Recovery as a journey of the heart. Psychiatric Rehabilitation Journal, 19, $\mathrm{n}$. 3, p. 91-97, Win 1996.

ONOCKO-CAMPOS, R. T.; PASSOS, E.; PALOMBINI ADE, L.; DOS SANTOS, D. V. et al. [Autonomous Medication Management: an analytical intervention in mental health care services]. Cien Saude Colet, 18, n. 10, p. 2889-2898, Oct 2013. 
RICCI; CRISTINA, E.; LEAL; MARIA, E. et al. Narratives about the Experience of Mental Illness: therecoveryProcess in Brazil. Psychiatric Quarterly, 92, n. 2, p. 573-585, Jun 2021.

RICCI, É. C.; LEAL, E.; LA-ROTTA, E. I. G.; ONOCKO-CAMPOS, R. et al. Cross-cultural adaptation of the recovery self-assessment instrument (RSA-R) person in recovery version to Brazilian Portuguese (Pt/Br). Journal of Public Mental Health, 2020.

SHANKAR, J.; LI, L.; TAN, S. Work Experiences and Challenges to Employment Sustainability for People With Mental Illness in Supported Employment Programs. Sage Open, 11, n. 3, Jul 2021. 